\title{
PULLBACK ATTRACTOR FOR A NON-AUTONOMOUS REACTION-DIFFUSION EQUATION IN SOME UNBOUNDED DOMAINS
}

\author{
MARÍA ANGUIANO \\ Dpto. Ecuaciones Diferenciales y Análisis Numérico \\ Universidad de Sevilla, Apdo. de Correos 1160, \\ 41080-Sevilla (Spain) \\ anguiano@us.es
}

\begin{abstract}
The existence of a pullback attractor in $L^{2}(\Omega)$ for the following nonautonomous reaction-diffusion equation

$$
\left\{\begin{array}{l}
\frac{\partial u}{\partial t}-\Delta u=f(u)+h(t), \text { in } \Omega \times(\tau,+\infty), \\
u=0, \text { on } \partial \Omega \times(\tau,+\infty), \\
u(x, \tau)=u_{\tau}(x), \quad x \in \Omega
\end{array}\right.
$$

is proved in this paper, when the domain $\Omega$ is not necessarily bounded but satisfying the Poincaré inequality, and $h \in L_{l o c}^{2}\left(\mathbb{R} ; H^{-1}(\Omega)\right)$. The main concept used in the proof is the asymptotic compactness of the process generated by the problem.
\end{abstract}

Key words: pullback attractor, asymptotic compactness, evolution process, non-autonomous reaction-diffusion equation.

AMS subject classifications: $\quad 35 B 41,35 Q 35,35 Q 30,35 K 90,37 L 30$.

\section{Introduction and setting of the problem}

Let $\Omega \subset \mathbb{R}^{N}$ be an open set, not necessarily bounded and suppose that $\Omega$ satisfies the Poincaré inequality, i.e., there exists a constant $\lambda_{1}>0$ such that

$$
\int_{\Omega}|u(x)|^{2} d x \leq \lambda_{1}^{-1} \int_{\Omega}|\nabla u(x)|^{2} d x, \quad \forall u \in H_{0}^{1}(\Omega) .
$$

Let us consider the following problem for a non-autonomous reactiondiffusion equation with zero Dirichlet boundary condition in $\Omega$,

$$
\left\{\begin{array}{l}
\frac{\partial u}{\partial t}-\Delta u=f(u)+h(t), \text { in } \Omega \times(\tau,+\infty), \\
u=0, \text { on } \partial \Omega \times(\tau,+\infty), \\
u(x, \tau)=u_{\tau}(x), \quad x \in \Omega,
\end{array}\right.
$$


where $\tau \in \mathbb{R}, u_{\tau} \in L^{2}(\Omega), h \in L_{l o c}^{2}\left(\mathbb{R} ; H^{-1}(\Omega)\right)$ and $f \in C(\mathbb{R})$ satisfies that there exist constants $\alpha_{1}>0, \alpha_{2}>0, l \geq 0$, and $p>2$ such that

$$
\begin{gathered}
-\alpha_{1}|s|^{p} \leq f(s) s \leq-\alpha_{2}|s|^{p}, \\
(f(s)-f(r))(s-r) \leq l(s-r)^{2} \quad \forall r, s \in \mathbb{R} .
\end{gathered}
$$

The aim of this paper is to show the existence of a pullback attractor in the phase space $L^{2}(\Omega)$ for the problem (3) in the case of open domains not necessarily bounded but satisfying the Poincaré inequality. This, and the fact that the nonautonomous $h$ belongs to the space $L_{l o c}^{2}\left(\mathbb{R} ; H^{-1}(\Omega)\right)$, are the main novelties of our problem.

The lack of compactness of the injection $H_{0}^{1}(\Omega) \subset L^{2}(\Omega)$ (in the case of unbounded domains) implies that the standard techniques previously used, particularly the one involving the so-called flatenning property (see [6], [7], [12], [14], amongst others), which have been successfully used when $\Omega$ is bounded and $h \in L_{\text {loc }}^{2}\left(\mathbb{R} ; L^{2}(\Omega)\right)$, do not work in our case.

Instead, we will use the asymptotic compactness already used in the case of non-autonomous 2D-Navier-Stokes (see [1] and [2], see also [5] for a close result), and which was previously used in [11] for the autonomous case. We would like to emphasize that this technique seems to be the only one which allows to prove the main result of this paper (namely Theorem 4) concerning the existence of pullback attractor for our problem.

It is also worth mentioning that our problem has received much attention over the last years in the case of a bounded domain or for a less general term $h$ (see [3], [7], [12], [14]).

Finally, the reader can find similar results for several variants of our model in the references [9], [10], among others.

\section{Existence and uniqueness of solution}

We state in this section a result on the existence and uniqueness of solution of problem (3). By $|\cdot|$ we denote the norm in $L^{2}(\Omega)$, by $|\nabla \cdot|$ the norm in $H_{0}^{1}(\Omega)$ and by $\|\cdot\|_{*}$ the norm in $H^{-1}(\Omega)$. We will use $(\cdot, \cdot)$ to denote the scalar product in $L^{2}(\Omega)$ and we will use $\langle\cdot, \cdot\rangle$ to denote the duality product between $H^{-1}(\Omega)$ and $H_{0}^{1}(\Omega)$.

Theorem 1 Suppose that $\Omega$ satisfies (2). Assume that $f \in C(\mathbb{R})$ satisfies (4) and (5), and $h \in L_{\text {loc }}^{2}\left(\mathbb{R} ; H^{-1}(\Omega)\right)$. Then, for all $\tau \in \mathbb{R}, u_{\tau} \in L^{2}(\Omega)$, there exists a unique solution $u(t)=u\left(t ; \tau, u_{\tau}\right)$ of (3) such that

$$
\begin{aligned}
& u \in L^{2}\left(\tau, T ; H_{0}^{1}(\Omega)\right) \cap L^{p}\left(\tau, T ; L^{p}(\Omega)\right) \quad \forall T>\tau, \\
& \frac{d}{d t}(u(t), v)-\langle\Delta u(t), v\rangle=\langle f(u(t)), v\rangle \\
& \quad+\langle h(t), v\rangle, \text { in } \mathcal{D}^{\prime}(\tau, \infty), \forall v \in H_{0}^{1}(\Omega) \cap L^{p}(\Omega), \\
& u(\tau)=u_{\tau} .
\end{aligned}
$$


Moreover,

$$
u \in C\left([\tau, \infty) ; L^{2}(\Omega)\right),
$$

and $u$ satisfies the energy equation,

$$
\begin{gathered}
\frac{1}{2} \frac{d}{d t}|u(t)|^{2}+|\nabla u(t)|^{2}=\langle f(u(t)), u(t)\rangle \\
+\langle h(t), u(t)\rangle \quad \text { in } \mathcal{D}^{\prime}(\tau, \infty) .
\end{gathered}
$$

Proof. The proof of this Theorem can be done by the method of monotony (see [8]).

\section{Preliminaries on the theory of pullback attractors}

Now, we will recall the main points from the theory of pullback attractors which will be needed to prove our objective (see [1] and [2] for more details).

Let us consider a process (also called a two-parameter semigroup) $U$ on a metric space $X$, i.e., a family $\{U(t, \tau) ;-\infty<\tau \leq t<+\infty\}$ of continuous mappings $U(t, \tau): X \rightarrow X$, such that $U(\tau, \tau) x=x$, and

$$
U(t, \tau)=U(t, r) U(r, \tau) \text { for all } \tau \leq r \leq t .
$$

Suppose that $\mathcal{D}$ is a nonempty class of parameterized sets $\widehat{D}=\{D(t) ; t \in \mathbb{R}\} \subset$ $\mathcal{P}(X)$, where $\mathcal{P}(X)$ denotes the family of all nonempty subsets of $X$.

Definition 1 The process $U(\cdot, \cdot)$ is said to be pullback $\mathcal{D}$-asymptotically compact if for any $t \in \mathbb{R}$, any $\widehat{D} \in \mathcal{D}$, any sequence $\tau_{n} \rightarrow-\infty$, and any sequence $x_{n} \in D\left(\tau_{n}\right)$, the sequence $\left\{U\left(t, \tau_{n}\right) x_{n}\right\}$ is relatively compact (i.e. pre-compact) in $X$.

Definition 2 It is said that $\widehat{B} \in \mathcal{D}$ is pullback $\mathcal{D}$-absorbing for the process $U(\cdot, \cdot)$ if for any $t \in \mathbb{R}$ and any $\widehat{D} \in \mathcal{D}$, there exists a $\tau_{0}(t, \widehat{D}) \leq t$ such that

$$
U(t, \tau) D(\tau) \subset B(t) \quad \text { for all } \tau \leq \tau_{0}(t, \widehat{D}) .
$$

Definition 3 The family $\widehat{A}=\{A(t) ; t \in \mathbb{R}\} \subset \mathcal{P}(X)$ is said to be a pullback $\mathcal{D}$-attractor for $U(\cdot, \cdot)$ if

1. $A(t)$ is compact for all $t \in \mathbb{R}$,

2. $\widehat{A}$ is pullback $\mathcal{D}$-attracting, i.e.,

$$
\lim _{\tau \rightarrow-\infty} \operatorname{dist}(U(t, \tau) D(\tau), A(t))=0,
$$

for all $\widehat{D} \in \mathcal{D}$, and all $t \in \mathbb{R}$,

3. $\widehat{A}$ is invariant, i.e.,

$$
U(t, \tau) A(\tau)=A(t), \quad \text { for }-\infty<\tau \leq t<+\infty .
$$


We have the following result (see [2] for more details).

Theorem 2 Suppose that the process $U(\cdot, \cdot)$ is pullback $\mathcal{D}$-asymptotically compact and that $\widehat{B} \in \mathcal{D}$ is a family of pullback $\mathcal{D}$-absorbing sets for $U(\cdot, \cdot)$.

Then, the family $\widehat{A}=\{A(t) ; t \in \mathbb{R}\} \subset \mathcal{P}(X)$ defined by $A(t)=\Lambda(\widehat{B}, t), \quad t \in$ $\mathbb{R}$, where for each $\widehat{D} \in \mathcal{D}$

$$
\Lambda(\widehat{D}, t)=\bigcap_{s \leq t}\left(\overline{\bigcup_{\tau \leq s} U(t, \tau) D(\tau)}\right),
$$

is a pullback $\mathcal{D}$-attractor for $U(\cdot, \cdot)$ which satisfies in addition that $A(t)=$ $\overline{\bigcup_{\widehat{D} \in \mathcal{D}} \Lambda(\widehat{D}, t)}$, for $t \in \mathbb{R}$. Furthemore, $\widehat{A}$ is minimal in the sense that if $\widehat{C}=\{C(t) ; \quad t \in \mathbb{R}\} \subset \mathcal{P}(X)$ is a family of closed sets such that $\lim _{\tau \rightarrow-\infty} \operatorname{dist}(U(t, \tau) B(\tau), C(t))=0$, then $A(t) \subset C(t)$.

\section{Existence of the pullback attractor}

Now, we can prove our main result in this paper. First, we need a continuity result which is established in the next subsection.

\subsection{Weak Continuity}

Assume that the function $f \in C(\mathbb{R})$ satisfies (4) and (5), and that $h \in$ $L_{l o c}^{2}\left(\mathbb{R} ; H^{-1}(\Omega)\right)$.

Thanks to Theorem 1 , we can define a process $\{U(t, \tau), \tau \leq t\}$ in $L^{2}(\Omega)$, as

$$
U(t, \tau) u_{\tau}=u\left(t ; \tau, u_{\tau}\right) \forall u_{\tau} \in L^{2}(\Omega), \forall \tau \leq t .
$$

From the uniqueness of solution to problem (3), it follows that (8) defines a process in $L^{2}(\Omega)$. In addition, it can be proved that the process defined by $(8)$ is continuous in $L^{2}(\Omega)$.

Moreover, $U$ is weakly continuous, and more exactly the following result holds true. We will denote by " $\rightarrow$ " the weak convergence in the corresponding indicated space, while " $\rightarrow$ " will denote the strong convergence, as usual.

Proposition 3 Let $\left\{u_{\tau_{n}}\right\} \subset L^{2}(\Omega)$ be a sequence converging weakly in $L^{2}(\Omega)$ to an element $u_{\tau} \in L^{2}(\Omega)$. Then, for all $T>\tau$, it follows

$$
\begin{gathered}
U(t, \tau) u_{\tau_{n}} \rightarrow U(t, \tau) u_{\tau} \text { in } L^{2}(\Omega) \forall t \geq \tau, \\
U(\cdot, \tau) u_{\tau_{n}} \rightarrow U(\cdot, \tau) u_{\tau} \text { in } L^{2}\left(\tau, T ; H_{0}^{1}(\Omega)\right), \\
U(\cdot, \tau) u_{\tau_{n}} \rightarrow U(\cdot, \tau) u_{\tau} \text { in } L^{p}\left(\tau, T ; L^{p}(\Omega)\right), \\
f\left(U(\cdot, \tau) u_{\tau_{n}}\right) \rightarrow f\left(U(\cdot, \tau) u_{\tau}\right) \text { in } L^{p \prime}\left(\tau, T ; L^{p^{\prime}}(\Omega)\right) .
\end{gathered}
$$

If $\Omega$ is a bounded set, then

$$
U(\cdot, \tau) u_{\tau_{n}} \longrightarrow U(\cdot, \tau) u_{\tau} \text { in } L^{2}\left(\tau, T ; L^{2}(\Omega)\right) .
$$


Proof. This result may be proved in much the same way as Theorem 1, and using similar arguments to [11].

\subsection{The existence of the global pullback attractor}

Let $\mathcal{R}_{\lambda_{1}}$ be the set of all functions $r: \mathbb{R} \rightarrow(0,+\infty)$ such that

$$
\lim _{t \rightarrow-\infty} e^{\lambda_{1} t} r^{2}(t)=0,
$$

and denote by $\mathcal{D}_{\lambda_{1}}$ the class of all families $\widehat{D}=\{D(t): t \in \mathbb{R}\} \subset \mathcal{P}\left(L^{2}(\Omega)\right)$ such that $D(t) \subset \bar{B}\left(0, r_{\widehat{D}}(t)\right)$, for some $r_{\widehat{D}} \in \mathcal{R}_{\lambda_{1}}$, where $\bar{B}\left(0, r_{\widehat{D}}(t)\right)$ denotes the closed ball in $L^{2}(\Omega)$ centered at zero with radius $r_{\widehat{D}}(t)$.

Now, we can prove the following result.

Theorem 4 Suppose that $\Omega$ satisfies (2), and suppose that $f \in C(\mathbb{R})$ satisfies (4) and (5) with $l=0$. Let $h \in L_{l o c}^{2}\left(\mathbb{R} ; H^{-1}(\Omega)\right)$ be such that

$$
\int_{-\infty}^{t} e^{\lambda_{1} s}\|h(s)\|_{H^{-1}(\Omega)}^{2} d s<+\infty \quad \forall t \in \mathbb{R} .
$$

Then, there exists a unique global pullback $\mathcal{D}_{\lambda_{1}}$-attractor for the process $U$, which belongs to $\mathcal{D}_{\lambda_{1}}$, and is defined by (8).

Proof. We only give the main ideas of the proof. Let $\tau \in \mathbb{R}$, and $u_{\tau} \in L^{2}(\Omega)$ be fixed, and denote

$$
u(t)=u\left(t ; \tau, u_{\tau}\right)=U(t, \tau) u_{\tau} \quad \forall t \geq \tau .
$$

Let $\widehat{D} \in \mathcal{D}_{\lambda_{1}}$ be given. Taking into account (2), (4), the energy equality and integrating between $\tau$ and $t$,

$$
\begin{aligned}
\left|U(t, \tau) u_{\tau}\right|^{2} \leq & e^{-\lambda_{1} t} \int_{-\infty}^{t} e^{\lambda_{1} s}\|h(s)\|_{H^{-1}(\Omega)}^{2} d s \\
& +e^{\lambda_{1}(\tau-t)} r_{D}^{2}(\tau),
\end{aligned}
$$

for all $u_{\tau} \in D(\tau)$ and for all $t \geq \tau$.

Denote by $R_{\lambda_{1}}(t)$ the nonnegative number given for each $t \in \mathbb{R}$ by

$$
R_{\lambda_{1}}^{2}(t)=e^{-\lambda_{1} t} \int_{-\infty}^{t} e^{\lambda_{1} s}\|h(s)\|_{H^{-1}(\Omega)}^{2} d s+1 .
$$

Observe that $R_{\lambda_{1}} \in \mathcal{R}_{\lambda_{1}}$. Now, consider the family $\widehat{B}_{\lambda_{1}}$ of closed balls in $L^{2}(\Omega)$, $\widehat{B}_{\lambda_{1}}=\left\{B_{\lambda_{1}}(t): t \in \mathbb{R}\right\}$, defined by $B_{\lambda_{1}}(t)=\left\{v \in L^{2}(\Omega):|v| \leq R_{\lambda_{1}}(t)\right\}$. It is straightforward to check that $\widehat{B}_{\lambda_{1}} \in \mathcal{D}_{\lambda_{1}}$, and moreover, by (14), the family $\widehat{B}_{\lambda_{1}}$ is pullback $\mathcal{D}_{\lambda_{1}}$-absorbing for the process $U$.

According to Theorem 2, to finish the proof of the theorem we only have to prove that $U$ is pullback $\mathcal{D}_{\lambda_{1}}$-asymptotically compact. 
Let us fix $\widehat{D} \in \mathcal{D}_{\lambda_{1}}$, a sequence $\tau_{n} \rightarrow-\infty$, a sequence $u_{\tau_{n}} \in D\left(\tau_{n}\right)$, and $t \in \mathbb{R}$. We have to prove that from the sequence $\left\{U\left(t, \tau_{n}\right) u_{\tau_{n}}\right\}$ we can extract a subsequence that converges in $L^{2}(\Omega)$.

As the family $\widehat{B}_{\lambda_{1}}$ is pullback $\mathcal{D}_{\lambda_{1}}$-absorbing, by a diagonal procedure, it is not difficult to conclude that there exist a subsequence $\left\{\left(\tau_{n^{\prime}}, u_{\tau_{n^{\prime}}}\right)\right\} \subset\left\{\left(\tau_{n}, u_{\tau_{n}}\right)\right\}$, and a sequence $\left\{w_{k} ; k \geq 0\right\} \subset L^{2}(\Omega)$ such that for all $k \geq 0$, and $w_{k} \in$ $B_{\lambda_{1}}(t-k)$,

$$
U\left(t-k, \tau_{n^{\prime}}\right) u_{\tau_{n^{\prime}}} \rightarrow w_{k} \text { in } L^{2}(\Omega),
$$

and

$$
\left|w_{0}\right| \leq \liminf _{n^{\prime} \rightarrow \infty}\left|U\left(t, \tau_{n^{\prime}}\right) u_{\tau_{n^{\prime}}}\right| .
$$

If we now prove that also

$$
\limsup _{n^{\prime} \rightarrow \infty}\left|U\left(t, \tau_{n^{\prime}}\right) u_{\tau_{n^{\prime}}}\right| \leq\left|w_{0}\right|,
$$

then we will have

$$
\lim _{n^{\prime} \rightarrow \infty}\left|U\left(t, \tau_{n^{\prime}}\right) u_{\tau_{n^{\prime}}}\right|=\left|w_{0}\right| .
$$

And this, together with the weak convergence, will imply the strong convergence in $L^{2}(\Omega)$ of $U\left(t, \tau_{n^{\prime}}\right) u_{\tau_{n^{\prime}}}$ to $w_{0}$.

In order to prove (18), consider $[u]:=|\nabla u|^{2}-\frac{\lambda_{1}}{2}|u|^{2}-\langle f(u), u\rangle$. Taking into account (2), (4), the energy equality and integrating between $\tau$ and $t$, it is immediate that for all $k \geq 0$ and all $\tau_{n^{\prime}} \leq t-k$,

$$
\begin{aligned}
& \left|U\left(t, \tau_{n^{\prime}}\right) u_{\tau_{n^{\prime}}}\right|^{2} \\
& =\left|U\left(t-k, \tau_{n^{\prime}}\right) u_{\tau_{n^{\prime}}}\right|^{2} e^{-\lambda_{1} k} \\
& +2 \int_{t-k}^{t} e^{\lambda_{1}(s-t)}\left\langle h(s), U(s, t-k) U\left(t-k, \tau_{n^{\prime}}\right) u_{\tau_{n^{\prime}}}\right\rangle d s \\
& -2 \int_{t-k}^{t} e^{\lambda_{1}(s-t)}\left[U(s, t-k) U\left(t-k, \tau_{n^{\prime}}\right) u_{\tau_{n^{\prime}}}\right] d s .
\end{aligned}
$$

Now we will prove that

$$
\begin{aligned}
& \int_{t-k}^{t} e^{\lambda_{1}(s-t)}\left[U(s, t-k) w_{k}\right] d s \\
& \leq \liminf _{n^{\prime} \rightarrow \infty} \int_{t-k}^{t} e^{\lambda_{1}(s-t)}\left[U(s, t-k) U\left(t-k, \tau_{n^{\prime}}\right) u_{\tau_{n^{\prime}}}\right] d s .
\end{aligned}
$$

Denote

$$
J_{k}(v)=J_{k}^{(1)}(v)+J_{k}^{(2)}(v)
$$

where

$$
J_{k}^{(1)}(v)=\int_{t-k}^{t} e^{\lambda_{1}(s-t)}\left(|\nabla v(s)|^{2}-\frac{\lambda_{1}}{2}|v(s)|^{2}\right) d s,
$$


and

$$
J_{k}^{(2)}(v)=-\int_{t-k}^{t} e^{\lambda_{1}(s-t)}\langle f(v), v\rangle d s,
$$

for all $v \in L^{2}\left(t-k, t ; H_{0}^{1}(\Omega)\right) \cap L^{p}\left(t-k, t ; L^{p}(\Omega)\right)$.

We also obtain from (16) and using Proposition 3

$$
\begin{aligned}
& \liminf _{n^{\prime} \rightarrow \infty}\left(J_{k}^{(1)}\left(U(\cdot, t-k) U\left(t-k, \tau_{n^{\prime}}\right) u_{\tau_{n^{\prime}}}\right)\right. \\
& \geq J_{k}^{(1)}\left(U(\cdot, t-k) w_{k}\right) .
\end{aligned}
$$

Using (5) with $l=0$, from (16) and Proposition 3 we easily obtain

$$
\begin{aligned}
& \lim _{n^{\prime} \rightarrow \infty} \inf \left(J_{k}^{(2)}\left(U(\cdot, t-k) U\left(t-k, \tau_{n^{\prime}}\right) u_{\tau_{n^{\prime}}}\right)\right) \\
& \quad \geq J_{k}^{(2)}\left(U(\cdot, t-k) w_{k}\right) .
\end{aligned}
$$

Therefore (20) is easily obtained from the last inequality and (21).

Then, taking into account that the family $\widehat{B}_{\lambda_{1}}$ is pullback $\mathcal{D}_{\lambda_{1}}$-absorbing, from (16), using Proposition 3 and thanks to (19) and (20), we obtain

$$
\begin{aligned}
& \lim _{n^{\prime} \rightarrow \infty} \sup \left|U\left(t, \tau_{n^{\prime}}\right) u_{\tau_{n^{\prime}}}\right|^{2} \\
& \quad \leq R_{\lambda_{1}}^{2}(t-k) e^{-\lambda_{1} k}+\left|w_{0}\right|^{2}
\end{aligned}
$$

for all $k \geq 1$. Taking into account (15), we easily obtain

$$
\lim _{n^{\prime} \rightarrow \infty} \sup \left|U\left(t, \tau_{n^{\prime}}\right) u_{\tau_{n^{\prime}}}\right|^{2} \leq\left|w_{0}\right|^{2}
$$

Acknowledgements. This work has been partially supported by Junta de Andalucía under project P07-FQM-02468.

\section{References}

[1] T. Caraballo, G. Lukaszewicz, and J. Real, Pullback attractors for asymptotically compact non-autonomous dynamical systems. Nonlinear Analysis, 64 (2006), 484-498.

[2] T. Caraballo, G. Lukaszewicz, and J. Real, Pullback attractors for nonautonomous 2D Navier-Stokes equations in unbounded domains. C. R. Math. Acad. Sci. Paris, 342 (2006), 263-268.

[3] T. Caraballo, J. A. Langa, and J. Valero, Dimension of attractors of nonautonomous reaction-diffusion equations. ANZIAM J., 45 (2003), 207222 .

[4] H. Crauel, A. Debussche, and F. Flandoli, Random attractors. J. Dyn. Diff. Eq., 9 (1995), no. 2, 307-341. 
[5] M.J. Garrido-Atienza and P. Marín-Rubio, Navier-Stokes equations with delays on unbounded domains. Nonlinear Anal., 64 (2006), no. 5, 11001118 .

[6] P.E. Kloeden and J.A. Langa, Flatenning, squeezing and the existence of random attractors. Proc. Roy. Soc. Lond. Series A, 463 (2007), 163-181.

[7] Y. Li and C.K. Zhong, Pullback attractors for the norm-to-weak continuous process and application to the nonautonomous reaction-diffusion equations. Applied Mathematics and Computation, 190 (2007), 1020-1029.

[8] J.L. Lions, Quelques Méthodes de Résolution des Problèmes aux Limites Non Linéaires. Dunod, Paris 1969.

[9] F. Morillas and J. Valero, Attractors for reaction-diffusion equations in $\mathbb{R}^{N}$ with continuous nonlinearity. Asymptotic Analysis, 44 (2005), 111-130.

[10] M. Prizzi, A remark on reaction-diffusion equations in unbounded domains. Discrete Contin. Dyn. Syst., 9 (2003), 281-286.

[11] R. Rosa, The Global Attractor for the 2D Navier-Stokes flow on some unbounded domains. Nonlinear Anal., 32 (1998), no. 1, 71-85.

[12] H. Song and H. Wu, Pullback attractors of nonautonomous reactiondiffusion equations. J. Math. Anal. Appl., 325 (2007), 1200-1215.

[13] R. Temam, Navier-Stokes equations and nonlinear functional analysis. CBMS-NSF Regional Conference Series in Applied Mathematics, Vol. 66. SIAM, Philadelphia, 1983 (2nd edition, 1995).

[14] Y. Wang and C. Zhong, On the existence of pullback attractors for nonautonomous reaction-difusion equations. Dynamical Systems, 23 (2008), $1-16$. 